\title{
A SIMULTANEOUS BIOGEOGRAPHY BASED OPTIMAL PLACEMENT OF DG UNITS AND CAPACITOR BANKS IN DISTRIBUTION SYSTEMS WITH NONLINEAR LOADS
}

\author{
Hassan Sadeghi — Navid Ghaffarzadeh *
}

\begin{abstract}
This paper uses a new algorithm namely biogeography based optimization (BBO) intended for the simultaneous placement of the distributed generation (DG) units and the capacitor banks in the distribution network. The procedure of optimization has been conducted in the presence of nonlinear loads (a cause of harmonic injection). The purpose of simultaneous optimal placement of the DG and the capacitor is the reduction of active and reactive losses. The difference in the values of loss reduction at different levels of the load have been included in the objective function and the considered objective function includes the constraints of voltage, size and the number of DG units and capacitor banks and the allowable range of the total harmonic distortion (THD) of the total voltage in accordance with the IEEE 519 standards. In this paper the placement has been performed on two load types ie constant and mixed power, moreover the effects of load models on the results and the effects of optimal placement on reduction of the THD levels have also been analyzed. The mentioned cases have been studied on a 33 bus radial distribution system.
\end{abstract}

K e y w or d s: biogeography based optimization, optimal placement, distributed generation units, capacitor banks, load models, harmonic load flow

\section{INTRODUCTION}

Distribution networks operate at low voltage and high current. Thus the level of losses is higher in the distribution networks as compared to the transmission networks which operates at a comparatively higher voltage. $13 \%$ of the produced power is lost in the distribution network [1]. High level of losses is the main cause of problems such as reduced equipment life, increased possibility of the power systems being unable in meeting the increasing demands of the customers and increase in the total cost for the customers.

Use of capacitor banks and distributed generation sources are one of the most effective ways for the reduction of losses. The optimal placement of DG in addition to reduction of active and reactive losses, improves voltage profile, increases reliability and network security and helps in lowering the harmonics and its adverse effects (such as the resonance phenomenon) [2-4]. Although the widespread use of DG units has affected the operation of the power system in the most desired way but still keeping in mind the limitations in size and power of the DG units (limited size of the DG units), its high installation and maintenance cost, these units can't be used on a much wider scale. Therefore the usage of the capacitor banks for improving the operation of the power system becomes quite necessary [2]. Capacitor banks are low in cost and it is easier to have an access to them, moreover the reactive power injected by parallel capacitors plays an effective role in reducing losses, improving the voltage profile, freeing up the system capacity and improving the power factor [5-7]. Therefore the simultaneous optimal placement of the capacitor banks and the DGs is quite important for acquiring optimization.

In [8-11] the reduction of losses has been considered as an important factor as far as the simultaneous placement of distributed generation units and capacitor banks is concerned. In [2] the improvement of voltage profile has been considered in addition to the reduction of the losses. [9-11] mentions the usage of sensitivity analysis in order to reduce the search space. In $[2,11,12]$ multi-level load has been taken into consideration and $[8,13]$ considers the load to be constant throughout the day. The research that has been carried out commonly uses a constant power load, so the dependence of the load on the bus voltage has been neglected $[14,15]$, this is despite the fact that in many real situations we have different types of load models such as the industrial, commercial and residential loads, these loads are closely associated with the bus voltage. Although the problem of harmonic pollution has been taken into account in different researches regarding the optimal placement of DG units and the optimal placement of capacitor banks $[16,17]$ but rarely any attention has been paid to the effect of the harmonics on the simultaneous placement of DG units and capacitor banks in several studies that have been done.

In order to optimize the placement problem, a variety of methods and algorithms have been used. In [2] the use of the hybridized algorithm ICA-GA has been proposed and its results has been compared with PSO. Reference [8] proposes the uses of BF-PSO algorithm and compares the results with GA, PSO, DE algorithms. [10,14] and [15] use the memetic, $\mathrm{ABC}$ and GA algorithms respectively.

* Technical and Engineering Faculty, Imam Khomeini International University, Qazvin, Iran, ghaffarzadeh@eng.ikiu.ac.ir 


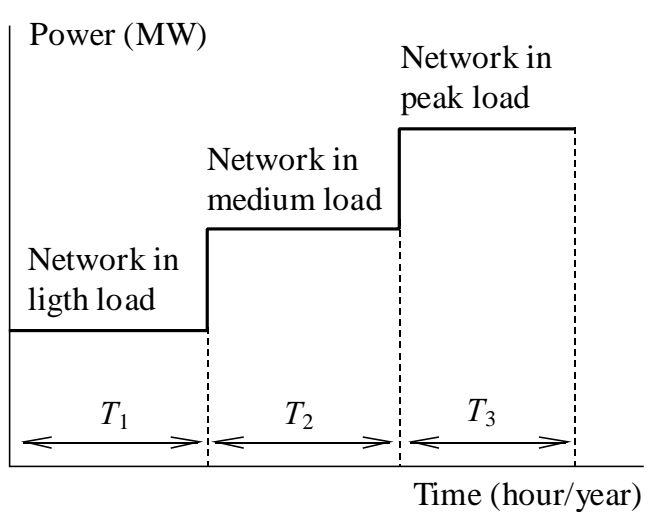

Fig. 1. Load models at three levels

Table 1. Values of $\alpha$ and $\beta$ for different load types

\begin{tabular}{ccc}
\hline Load type & $\alpha$ & $\beta$ \\
\hline Constant power & 0 & 0 \\
Industrial & 6 & 0.18 \\
Commercial & 3.4 & 1.51 \\
Residential & 4.04 & 0.92 \\
\hline
\end{tabular}

In this paper, the optimal location and size of the capacitor banks and DG units has been specified according to the harmonic pollution of the specified network. Nonlinear loads are the sources of harmonic injection. All the network loads are considered as multilevel ones and the optimization procedure is done for two load models, one being the constant load models and other one is the combinational load comprising of commercial, industrial and the residential loads. A new algorithm namely biogeography based optimization (BBO) has been used for the reduction of active and reactive losses and the value of loss reduction in the various load levels has been taking into account. The proposed algorithm has been implemented in MATLAB and harmonic load flow has been analyzed using the DIgSILENT software.

\section{LOAD MODEL}

The correct calculation and analyses of the objective function requires correct and true input values and errorless calculations performed on the given input information. One of the most important input information is about the load model. This paper mentions the daily changes in network load distribution modeled at three different levels (ie light load, medium load and peak load). Figure 1 shows the mentioned model.

In different studies, usually the load model has been assumed to be a constant power model, while in reality the networks loads are mixed loads whose active and reactive power depends upon the bus voltage. In this paper in addition to the constant power load model, load models comprising of industrial, commercial and residential loads have also been analyzed [18]. The dependence of the active and reactive loads on the voltage magnitude has been shown using

$$
P=P_{0} V^{\alpha}, \quad Q=Q_{0} V^{\beta},
$$

where $P$ and $Q$ are the active and reactive power respectively; $P_{0}$ and $Q_{0}$ are the active and reactive powers for the rated voltage respectively; $\alpha$ and $\beta$ show the power of the voltage for the active and reactive power; moreover their values have been given in Tab. 1 for different types of loads.

\section{RELATIONS GOVERNING THE PROBLEM}

The simultaneous optimal placement of DG units and capacitor banks for the reduction of active and reactive loads has been done with the problem boundaries kept in mind which would be discussed as we progress.

\subsection{The Objective Function}

The difference between the reduction of the value of active and reactive losses at different levels of load has been considered in the purposed objective function

$$
\begin{aligned}
O F=\omega_{1}\left(\sigma_{1} f_{P L}+\sigma_{2} f_{Q L}\right) & +\omega_{2}\left(\sigma_{1} f_{P M}+\sigma_{2} f_{Q M}\right) \\
& +\omega_{3}\left(\sigma_{1} f_{P H}+\sigma_{2} f_{Q H}\right) .
\end{aligned}
$$

In the above equation $f_{P L}, f_{P M}$ and $f_{P H}$ represent the active losses at light, medium and peak load levels respectively. $f_{Q L}, f_{Q M}$ and $f_{Q H}$ represent the reactive losses at the light, medium and peak levels of load. $\omega_{1}=$ $0.2, \omega_{2}=0.35$ and $\omega_{3}=0.45$ are the coefficients of the objective function at the light, medium and peak levels of load respectively. $\sigma_{1}=0.6$ and $\sigma_{2}=0.4$ are the coefficients of the reduction indicators of the active and reactive losses. Coefficients have specified according to the importance of every section of the objective function and follow the relationships given below.

$$
\sum_{i=1}^{n} \omega_{i}=1, \quad \sum_{j=1}^{m} \sigma_{j}=1 .
$$

In the above equations $\mathrm{n}$ represents the number of load levels under consideration and $\mathrm{m}$ is the number of indicators that have been included in every load level.

\section{Active and Reactive Power Loss Indices}

The active and reactive power loss indices can be calculated by using the following equations.

$$
f_{P}=\frac{P_{L w}}{P_{L n}}, \quad f_{Q}=\frac{Q_{L w}}{Q_{L n}} .
$$

$P_{L w}$ and $P_{L n}$ represent the level of active losses before and after the installation of the equipment respectively, similarly $Q_{L w}$ and $Q_{L n}$ represent the level of reactive losses before and after the installation of the equipment, respectively. The mentioned quantities are calculable for all the load levels. 
Table 2. Harmonic percentage of the six pulse converter

\begin{tabular}{c|ccccccc}
\hline Harmonic order & 5 & 7 & 11 & 13 & 17 & 19 & 23 \\
\hline Harmonic current (\%) & 20 & 14.3 & 9.1 & 7.7 & 5.9 & 4 & 3.4 \\
\hline
\end{tabular}

\subsection{Problem Constraints}

\section{Voltage level Constraints}

Voltage of each bus must be within the allowed range according to the following equation

$$
v_{\min } \leq \sqrt{\left(v_{i}^{(1)}\right)^{2}+\sum_{h=2}^{h_{\max }}\left|v_{i}^{h}\right|^{2}} \leq v_{\max }
$$

In the above mentioned equation $v_{\min }=0.95$ and $v_{\max }=$ 1.05 are the minimum and maximum permissible values of the effective voltage of every bus respectively. $v_{i}$ is the fundamental voltage of the $i^{\text {th }}$ bus and $h$ is the harmonic order.

\section{Harmonic constraints}

According to IEEE-519 standards, total harmonic distortion (THD) of the voltage of every bus should be less than $5 \%$. These constraints can be achieved using the following equation

$$
\operatorname{TH}_{v, i}(\%)=\frac{\sqrt{\sum_{h=2}^{h_{\max }}\left|v_{i}^{h}\right|}}{\left|v_{i}\right|} \leq T H D_{v}^{\max }
$$

Where $T H D_{v}^{\max }$ is equal to $5 \%$.

Generated power constraints of the distributed generation units

According to the equations given below (equations 10 and 11) the active power and the power factor of the DG units lies between a maximum and a minimum value.

$$
\begin{aligned}
P_{D G} & =\left[0, P_{D G}^{\max }\right], \\
f_{D G} & =\left[0, p f_{D G}^{\max }\right] .
\end{aligned}
$$

Also the total power generated by the DGs should be less than the total power load of the network:

$$
\sum_{i=1}^{n} \sqrt{\left(P_{i}^{D G}\right)^{2}+\left(Q_{i}^{D G}\right)^{2}}<\sum_{i=1}^{n} \sqrt{\left(P_{i}^{L}\right)^{2}+\left(Q_{i}^{L}\right)^{2}} .
$$

$P_{i}^{L}$ and $Q_{i}^{L}$ are the active and the reactive power loads of the $i^{\text {th }}$ bus whereas $P_{i}^{D G}$ and $Q_{i}^{D G}$ are the active and reactive power generated by the DGs in the $i^{\text {th }}$ bus.
Parallel capacitors size constraints

Capacitors available in the industry have specified sizes. Discrete capacitors and capacitors of the following sizes; 0.15, 0.3, 0.45, 0.6, 0.75, 0.9, 1.05 (MVar) have been used in this paper for optimization and the optimal capacitor value for each bus has been selected from the capacitors mentioned above [16].

\section{BIOGEOGRAPHY-BASED OPTIMIZATION ALGORITHM}

Biogeography-based optimization (BBO) is a new global optimization algorithm based on the theory of biogeography which deals with the geographical distribution of biological organisms. The migration of species from one island to the other, the emergence of new species and the extinction of a species is described by the mathematical models of biogeography. Those geographic areas which are a good place to live for the species have a high habitat suitability index. Precipitation level, vegetation density, arid areas and temperature are the characteristics associated with HSI. Variables that shape this suitability are known as Suitability index variables. SIVs can be considered as variables independent of the habitat and HSI. A good solution ie an island with a high HSI and similarly a weak solution ie an island with a low HSI have been mentioned below. Sharing of information in this method is done using migration operator. Solutions with a high HSI tend to show interest in sharing characteristics with solutions having a weak HIS [19].

\subsection{The steps implementation of the BBO algo- rithm}

The two other important concepts in this algorithm are the "migration" and the "Immigration" capabilities also $\mu$ and $\lambda$ represent the amounts of the above mentioned concepts at any particular location respectively. The steps implementation of the BBO algorithm have been mentioned below and its flowchart has been shown in Fig. 2.

1) Creation of initial population.

2) Determining $\mu$ and $\lambda$ in terms of ranking.

3) Implementing steps 4 to 8 for every residential area $i$.

4) Implementing steps 5 to 8 for the variable $\mathrm{k}$ of residential area $i$.

5) Making changes in variable $\mathrm{k}$ with the probability of $\lambda$.

6) Migrating by the interference of $\mu$ and $\lambda$.

7) Making random change (mutation) on variable.

8) Assessing the newly obtained population.

9) Composing initial population and obtained population.

10) Checking final condition. 


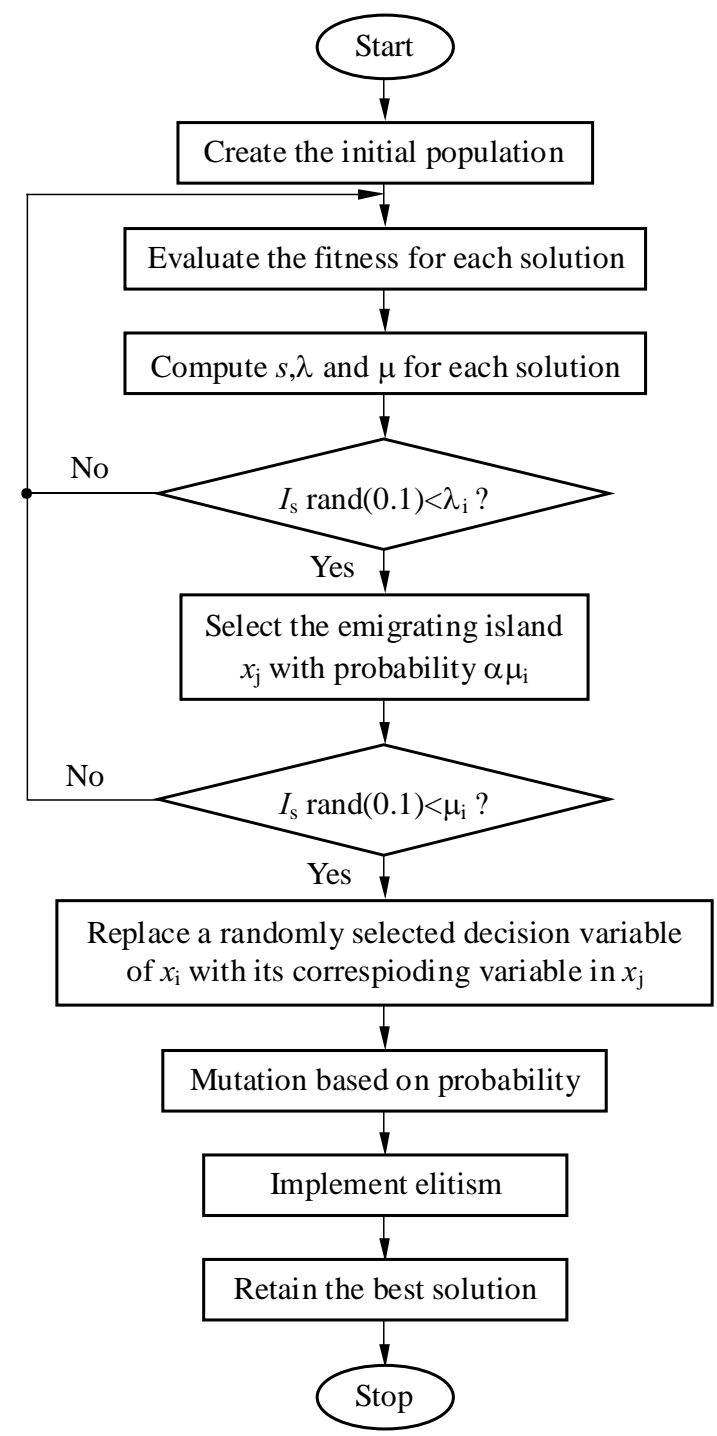

Fig. 2. BBO algorithm flowchart

The science of biogeography on which BBO is the study of biological organism based on geographical distribution. The migration patterns of the organisms and the process of the extinction as well as existence is studied using bio-geography.

Biogeography introduces couple of new terms such as HSI and SIBs. HSI is the abbreviated form of Habitats suitability index which signifies the suitability of the habitat for living that is higher the HSI the more the area is suitable for the biological species as a habitat. Rate of pre- cipitation, topography of the area, temperature etcare the features that determine the HSI. The above mentioned features are characterized by a set of variables known as SIVs, so it can be said that the SIVs are the independent variables of a particular habitat where as HSIs are the dependent ones. HSI widely determines the population of the habitat $i e$ if HSI is on a higher side the habitat would have a high population and vice versa. Emigration and immigration are the two fundamental concepts that are used in BBO. Relations governing these concepts are

$$
\begin{aligned}
& \mu_{s}=E\left(\frac{S}{S_{\max }}\right), \\
& \lambda_{s}=I\left(1-\frac{S}{S_{\max }}\right) .
\end{aligned}
$$

In the above mentioned relations, $I$ determines the maximum possible immigration rate when the number of species on the inland (habitat) is zero and $E$ signifies the maximum possible emigration rate.

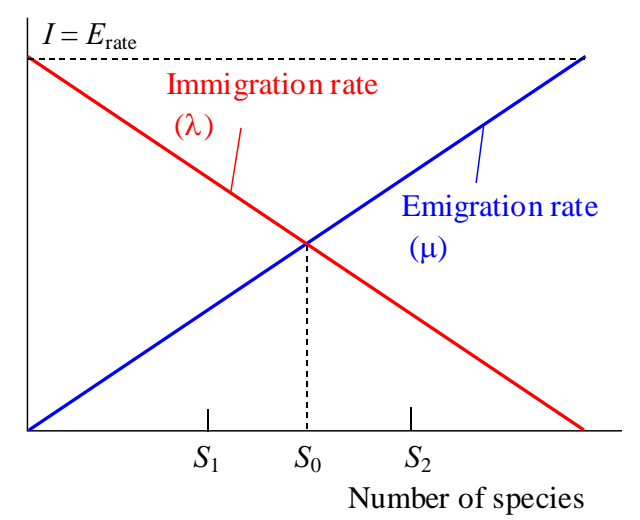

Fig. 3. The relationship between the fitness of habitats (number of species), emigration rate $\mu$ and immigration rate $\lambda$

In Fig. 3, the relationships between $\mu$ and $\lambda$ (emigration and immigration rates respectively) have been shown. $S_{0}$ is the equilibrium species number. It is the point of interaction of the immigration rate of the new species and the extinction of the older ones. When $S=$ $S_{0}$, emigration becomes equal to the immigration.

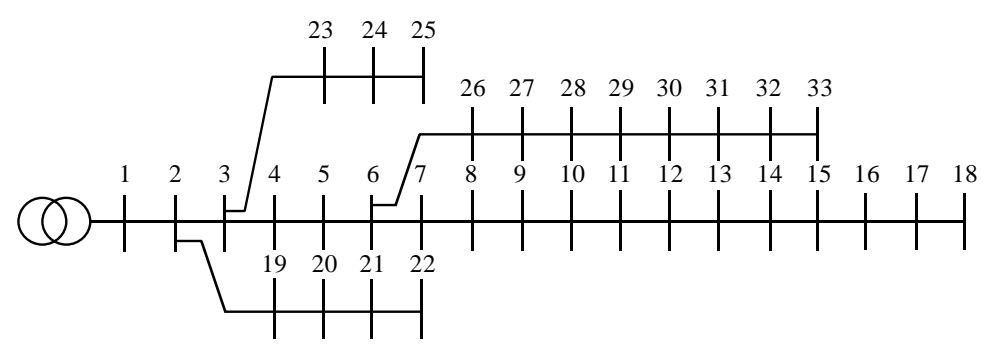

Fig. 4. 33 bus radial distribution network 
Table 3. The location and size of DG units and capacitors for two different load models

\begin{tabular}{cccccc}
\hline Load model & $\begin{array}{c}\text { Optimal placement } \\
\text { bus for capacitor }\end{array}$ & $\begin{array}{c}\text { Capacitor } \\
\text { size (Mvar) }\end{array}$ & $\begin{array}{c}\text { Optimal } \\
\text { placement } \\
\text { bus for } \\
\text { capacitor }\end{array}$ & $\begin{array}{c}\text { DG size } \\
(\mathrm{MW})\end{array}$ & $\begin{array}{c}\text { DG Power } \\
\text { Factor }\end{array}$ \\
\hline & $5,8,9,14,15,28,31$ & 0.15 & 3 & 1.346 & 0.82 \\
Constant load & 1,10 & 0.30 & 7 & 1.511 & 0.99 \\
& 16,27 & 0.45 & 25 & 0.640 & 0.99 \\
\hline Combination & 33 & 0.60 & - & - & - \\
of industrial & $6,26,32$ & 0.90 & - & - & 0.917 \\
commercial & $9,14,20,25$ & 0.15 & 3 & 1.088 & 0.99 \\
and residential & 16,32 & 0.30 & 26 & 1.486 & 0.99 \\
loads & $28,29,31$ & 0.45 & 27 & - & - \\
\hline
\end{tabular}

\section{A TEST NETWORK AND SIMULATION RESULTS}

\subsection{Test network}

The proposed method for the simultaneous optimal placement of DG units and the capacitors has been tested on a common sample network with 33 buses having a rated voltage of $12.6 \mathrm{KV}$. Single-line diagram of the network has been shown in Fig. 4. Two nonlinear loads have been placed on the buses 5,26 . The nonlinear loads are in the form of a six-pulse converter with the active and reactive power equal to $1 \mathrm{MW}$ and are $0.75 \mathrm{MVar}$. The Percentage of the harmonic current injection of the sixpulse converter has been given in Tab. 2 .

In this paper the maximum number of DG units is 3 and the maximum number of the capacitors has been taken equal to the number of buses of the test network. $P_{D G}^{\max }=2 \mathrm{MW}, p f_{D G}^{\max }=1$ and the maximum output power of all DG units has been considered equal to $3.5 \mathrm{MW}$.

\subsection{Simulation results}

The main purpose of this article is to find out the location and size of capacitor banks and DG units simultaneously and to reduce the active and reactive power losses at different load levels.

Optimal size and location of the DG units and capacitor banks

The optimal size and location of the DG units and the capacitor banks for constant power load models and mixed load models ( $i e$ the combination of industrial, commercial and residential loads) has been shown in Tab. 3 . As it is quite evident, with change in the type of load, the optimal size and location of the capacitors and the DG units experience a drastic change.
The impact of the simultaneous optimized placement of DG and capacitor on performance of the system

The rate of losses of the distribution network is dependent on the location and the size of the capacitor and DG present in the network, therefore their proper placement reduces the power losses. Table 4 shows the amount of the reactive and active losses in the network based on their type (constant and mixed) and also based on three load levels (low, medium and peak). It is evident that the optimal placement reduces the active and reactive power losses at different load levels significantly. The optimal placement of DG and Capacitors can also be used to reduce network harmonic pollution significantly. Table 4 shows the maximum amount of THD of the network both before and after optimization.

Also, Fig. 5 shows the level of THD of the voltage of all buses at peak load level. The level of THD of the network before optimization was outside the acceptable limit but after the installation of the optimization instruments the THD level was reduced and its level came within the acceptable range (in accordance with the IEEE-519 standards).

One of the most important effects of specification of the optimal location and size of DG units and the capacitors is the improvement in voltage profile. Table 4 shows the lowest effective voltage at peak load level and Fig. 6 shows the voltage profile at the peak load level and at two load models ie constant and mixed load models, according to the Tab. 4 and Fig. 6, the improvement in the voltage profile is due to the optimization.

\section{CONCLUSION}

In this paper, a new algorithm namely biogeography based optimization (BBO) has been used in order to reduce the active and reactive losses and to take into account the value reduction of losses at different load levels. The results obtained show that the simultaneous placement of the DG units and capacitors in addition to reduction of losses and improvement of the voltage profile can 

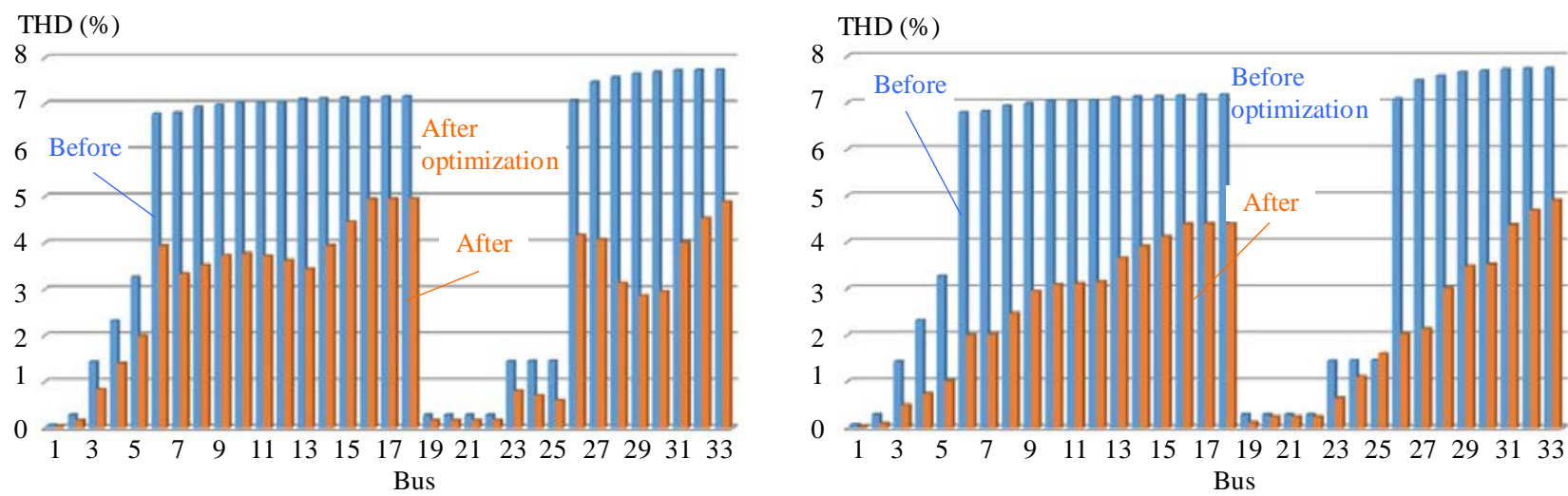

Fig. 5. THD of the voltage at peak load level (1) constant power load (2) mixed load
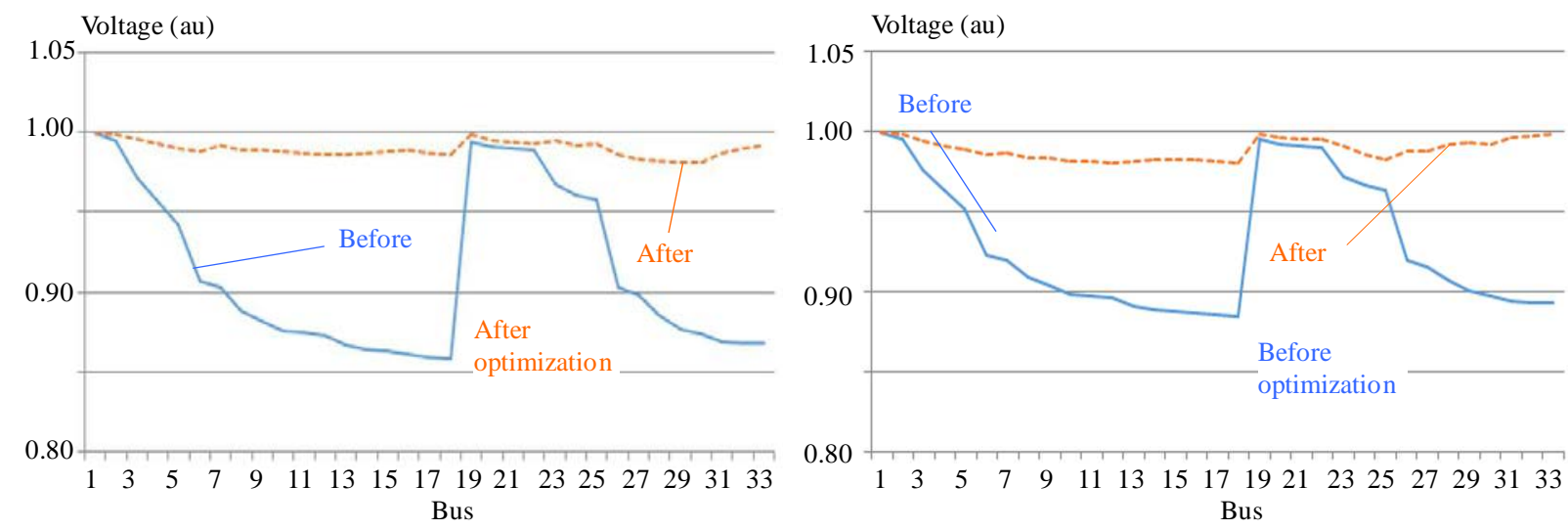

Fig. 6. The voltage profile at peak load level (1) constant power load (2) mixed load

Table 4. The results obtained after simultaneous placement of DG units and capacitor banks

\begin{tabular}{|c|c|c|c|c|c|c|}
\hline \multirow[b]{2}{*}{ Load model } & \multirow[b]{2}{*}{ Status } & \multicolumn{3}{|c|}{ Power Losses(MW + MVar) } & \multirow{2}{*}{$\begin{array}{l}\text { The lowest } \\
\text { amount of } \\
\text { effective } \\
\text { voltage at } \\
(\mathrm{pu})\end{array}$} & \multirow{2}{*}{$\begin{array}{c}\text { The highest } \\
\text { amount of } \\
\text { THD at } \\
\text { peak load } \\
(\%)\end{array}$} \\
\hline & & $\begin{array}{l}\text { At light load } \\
\text { level }\end{array}$ & $\begin{array}{l}\text { At medium } \\
\text { load level }\end{array}$ & $\begin{array}{l}\text { At peak load } \\
\text { level }\end{array}$ & & \\
\hline \multirow[b]{2}{*}{$\begin{array}{l}\text { Constant } \\
\text { load }\end{array}$} & $\begin{array}{c}\text { Before } \\
\text { optimization }\end{array}$ & $0.356+j 0.215$ & $0.462+j 0.278$ & $0.588+j 0.351$ & 0.86 & 7.74 \\
\hline & $\begin{array}{c}\text { After } \\
\text { optimization }\end{array}$ & $0.279+j 0.200$ & $0.265+j 0.190$ & $0.264+j 0.187$ & 0.98 & 4.96 \\
\hline \multirow{2}{*}{$\begin{array}{l}\text { Combination } \\
\text { of industrial, } \\
\text { commercial and } \\
\text { residential loads }\end{array}$} & $\begin{array}{c}\text { Before } \\
\text { optimization }\end{array}$ & $0.262+j 0.156$ & $0.329+j 0.193$ & $0.402+j 0.234$ & 0.88 & 7.74 \\
\hline & $\begin{array}{c}\text { After } \\
\text { optimization }\end{array}$ & $0.133+j 0.104$ & $0.135+j 0.105$ & $0.146+j 0.111$ & 0.98 & 4.90 \\
\hline
\end{tabular}

also be used to reduce the harmonic pollution of the network thus bringing it inside the acceptable range. Also in this paper the optimal size and location of DG units and capacitors at constant power load model and mixed load models ie (industrial, commercial and residential) related to the voltage has also been studied. It was observed that load model has a significant effect on the location and the size of DG units and capacitors, the amount of active and reactive losses and the voltage level. The results show that load models didn't have any effect on the THD of the voltage buses before optimization but after the optimization, load models had such an effect on the location and the size of the DG units and the capacitor that the THD of the voltage buses is completely different at these two load models.

\section{REFERENCES}

[1] GOPIYA NAIK, S.-KHATOD, D. K.-SHARMA, M. P. : Optimal Allocation of Combined DG and Capacitor for Real Power 
Loss Minimization in Distribution Networks, International Journal of Electrical Power and Energy Systems 53 (2013), 967-973.

[2] MORADI, M. H.-ZEINALZADEH, A.-MOHAMMADI, Y. -ABEDINI, M. : An Efficient Hybrid Method for Solving the Optimal Sitting and Sizing Problem of DG and Shunt Capacitor Banks Simultaneously based on Imperialist Competitive Algorithm and Genetic Algorithm, International Journal of Electrical Power and Energy Systems 54 (2014), 101-111.

[3] HEDAYATI, H.-NABAVINIAKI, SA.-AKBARIMAJD, A. : A Method for Placement of DG Units in Distribution Networks, IEEE Transactions on Power Delivery 23, No. 3 (2008), 1620-1628.

[4] BISWAS, S.-GOSWAMI, S. K.-CHATTERJEE, A. : Optimum Distributed Generation Placement with Voltage Sag Effect Minimization, Energy Conversion and Management 53 (2012), 163-174.

[5] TAHER, S. A.-BAGHERPOUR, R.: A New Approach for Optimal Capacitor Placement and Sizing in Unbalanced Distorted Distribution Systems Using Hybrid Honey Bee Colony Algorithm, International Journal of Electrical Power and Energy Systems 49 (2013), 430-448.

[6] ZIARI, I.-LEDWICH, G.-GHOSH, A.-CORNFORTH, D. -WISHART, M. : Optimal Allocation and Sizing of Capacitors to Minimize the Transmission Line Loss and to Improve the Voltage Profile, Computers and Mathematics with Applications 60 (2010), 1003-1013.

[7] XIN-MEI YU-XIN-YIN XIONG-YAO-WU WU: A PSOBased Approach to Optimal Capacitor Placement with Harmonic Distortion Consideration, Electric Power Systems Research 71 (2004), 27-33.

[8] MORADI, M. H.-ABEDINI, M. : A Combination of Genetic Algorithm and Particle Swarm Optimization for Optimal DG Location and Sizing in Distribution Systems, International Journal of Electrical Power and Energy Systems 34 (2012), 66-74.

[9] HOOSHMAND, R. A.-MOHKAMI, H. : New Optimal Placement of Capacitors and Dispersed Generators Using Bacterial Foraging Oriented by Particle Swarm Optimization Algorithm in Distribution Systems, Electrical Engineering 93 (2011), 43-53.

[10] SAJJADI, S. M.-HAGHIFAM, M. R.-SALEHI, J. : Simultaneous Placement of Distributed Generation and Capacitors in Distribution Networks Considering Voltage Stability Index, International Journal of Electrical Power and Energy Systems 46 (2013), 366-375.

[11] GOPIYA NAIK, S.-KHATOD, D. K.-SHARMA, M. P. : Optimal Allocation of Combined DG and Capacitor for Real Power Loss Minimization in Distribution Networks, International Journal of Electrical Power and Energy Systems 53 (2013), 967-973.

[12] BASUONY, E.-ABDEL-SALAM, T. S.-ATTIA, A. S.BADR, M. A. : Power System Efficiency and Voltage Regulation as Affected by Dispersed Generation, 39th International Universities Power Engineering Conference, vol. 3, 2004, pp. 1337-1341.

[13] ABDI, S.-AFSHAR, K. : Application of IPSO-Monte Carlo for Optimal Distributed Generation Allocation and Sizing, International Journal of Electrical Power and Energy Systems 44 (2013), 786-797.

[14] ABU-MOUTI, F. S.-El-HAWARY, M. E.: Optimal Distributed Generation Allocation and Sizing in Distribution Systems Using Artificial Bee Colony Algorithm, IEEE Transactions on Power Delivery 26 No. 4 (2011), 2090-2101.

[15] MithulanATHAN, N.-OO THAN-VAN PHU LEE : Distributed Generator Placement in Power Distribution System Using Genetic Algorithm to Reduce Losses, Thammasat International Journal of Science and Technology 9 No. 3 (2004), 55-62.

16] TAHER, S. A.-HASANI, M.-KARIMIAN, A.: A Novel Method for Optimal Capacitor Placement and Sizing in Distribution Systems with Nonlinear Loads and DG using GA, Communication in Nonlinear Science and Numerical Simulation 16 (2011), 851-862.

[17] RAVIKUMAR PANDI, V.-ZEINELDIN, H. H.-WEIDONG XIAO-ZOBAA, A. F.: Optimal Penetration Levels for Inverter-Based Distributed Generation Considering Harmonic Limits, Electric Power Systems Research 97 (2013), 68-75.

18] SINGH, D.-SINGH, D.-VERMA, K. S. : Multi Objective Optimization for DG Planning with Load Models, IEEE Transactions on Power Systems 24 No. 1 (2009), 427-36.

19] SIMON, D. : Biogeography-Based Optimization, IEEE Transactions on Evolutionary Computation 12 No. 6 (2008), 702-713.

Received 26 June 2015

Hassan Sadeghi, received MS degree in electrical engineering from the Imam Khomeini International University, Qazvin, Iran. His areas of research interest include power system optimization and power quality.

Navid Ghaffarzadeh (Dr) is an assistant professor of electrical engineering at Imam Khomeini International University. He received MS and $\mathrm{PhD}$ degrees in electrical engineering from the Amirkabir University of Technology (graduated with First Class Honors), Tehran, Iran, and the Iran University of Science and Technology (graduated with First Class Honors), Tehran, Iran, respectively. His research interests include power systems protection and control, transient in power systems and power quality. He is the author of three books in the field of power systems. He is also the author and the coauthor of 74 technical papers. 